\title{
Bi-axial Tensile Behavior of Woven Fabrics Sewn with Open Lock Stitches to Form Cylinder
}

\author{
By Tsuneo Horino* and Sadako Kawanishi**, Members, TMSJ \\ *Department of Textile Engineering, Art Industry and Textile College of Kyoto, Kyoto \\ **Department of Clothing Science, Mukogawa Women's University, Nishinomiya, Hyogo-ken
}

Based on The Journal of the Textile Machinery Society of Japan, Transactions, Vol. 26, No. 9, T156-164 (1973)

\begin{abstract}
Bi-axial tensile behavior of woven fabrics sewn with open lock stitches was observed by using an Instron type tensile tester with a simple attachment made of a Teflon rod.

Specimens were made into circular cylinders by sewing along warps or wefts. The models mechanically equivalent to these sewn specimens were assumed by the same way as explained in the previous paper ${ }^{[1]}$.

The experimental results were analysed on the assumption that the seam element consists of six straight threads, and when stretched, these yarns are extended with no slip against the fabrics into which the threads are sewn in.

The analysis gives that it is necessary to increase the seam strength by balancing both tensions of the needle thread and the bobbin thread.
\end{abstract}

\section{Introduction}

In the previous paper ${ }^{[1]}$, the tensile behavior of woven fabrics sewn with open-lock stitches was studied when they were stretched in the direction perpendicular to the seam line and analysed by considering a mechanical model in which the fabric element and the seam element were connected in series. In this case, because the stitch interval decreased when the specimen was stretched, the analysis of its tensile behavior was limited within the low load region in which the stitch interval might be thought to keep the original interval.

In this paper, a simple attachment was used in order to keep the original stitch interval constant even when the specimen was subjected to a tensile strain in both directions parallel and perpendicular to the seam line, and the tensile experiment of the sewn specimens was carried out by using a usual tensile tester. The results obtained were analysed by the way as in the previous paper ${ }^{[1]}$.

\section{Tensile Experimental Method}

In order to measure the tensile behavior of sewn specimens while keeping their width constant, an attachment shown in Fig. 1 was used, which consists of two connecting screw rods (1) and (5), two stepped rods (2) and (4), and a Teflon rod (3). Screw rods are mounted on upper and lower clamps of an Instron type tensile tester, respectively.

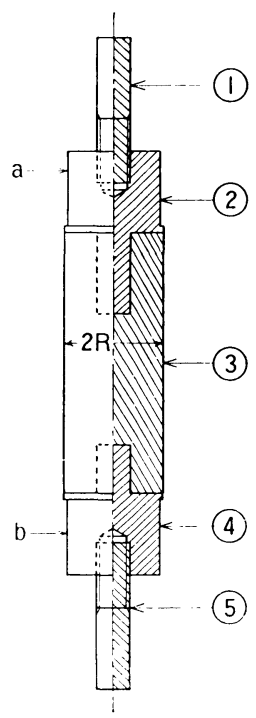

Fig. 1 A simple attachment used, (1), (5); connecting screw rods; (3): Teflon rod; (2), (4): stepped rod

A sewn specimen having the peripheral length as long as the circumference of the Teflon rod was put on this rod, and its gauge length was determined by lifting the cross head of the tester. Then the specimen was clamped at (a) and (b) shown in Fig. 1 with steel bands and thick paper. 




Fig. 2 A sewn specimen put on the attachment

In order to reduce the frictional resistance between the cylindrical test specimen and rod (3), such a smooth rod was used as was made of Teflon.

Fig. 2 shows the sewn specimen on the attachment and ready to be examined in bi-axial testing. Although the circumference of the cylindrical specimen tends to become short when stretched in the direction of the rod axis, we kept it constant by inserting rod (3). Namely, when the sewn specimen is stretched along the rod axis, its circumferencial strain is kept zero.

\section{Analysis of Bi-axial Tensile Behavior}

Consider the case in which a cylindrically sewn specimen as shown in Fig. 3(a) is subjected to longitudinal strain. The seam line is made along the warp or the weft direction of the fabric to form a generating line on the cylinder. The circumference of the specimen $L_{X, s}$ is given by:

$$
L_{X, S}=L_{X, f}+L_{X, y}=\pi(2 R+t)
$$

where $L_{X, f}$ : length of a fabric element in $\mathrm{X}$ direction

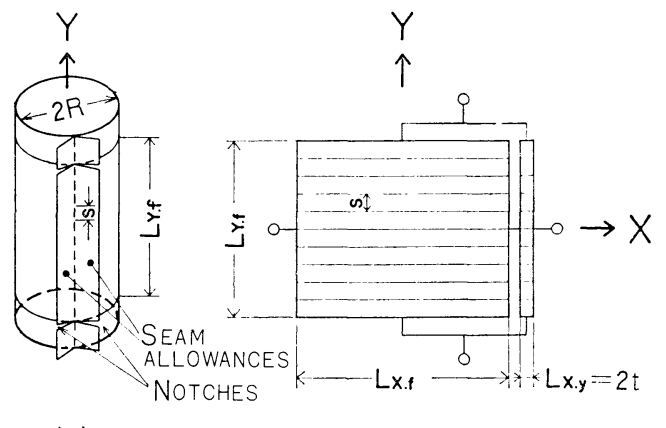

(a)

(b)

Fig. 3 (a) Cylindrical by sewn specimen having a seam line

(b) Equivalent mechanical model
$L_{X, y}$ : length of a seam element in X direction

$R$ : radius of rod (3)

$t$ : thickness of the seam element and $t \ll R$

If the specimen is sewn in a cylindrical shape with a uniform stitch length $s$, the gauge length of the test specimen $L_{Y, f}$ shown in Fig. 3(a) is equal to $n \times s$. When an equivalent mechanical model of Fig. 3(a) is derived by the same way as in the previous paper ${ }^{[1]}$, it is expressed by Fig. 3(b). To exclude the influence of the seam allowances, the end parts were cut with a pair of scissors as shown in Fig. 3(a).

The strain $\varepsilon_{X, s}$ in $\mathrm{X}$ direction may be written by the following equations as in previous paper ${ }^{[1]}$, assuming that strains $\varepsilon_{X, f}$ and $\varepsilon_{X, y}$ have the simple additive property.

$$
\begin{aligned}
& P_{X, S}=P_{X}, f=P_{X, y}(t) \\
& \varepsilon_{X}, S=\phi_{f} \circ \varepsilon_{X}, f+\phi_{y} \circ \varepsilon_{X}, y(i) \\
& \phi_{f}=L_{X}, f / L_{X}, s, \phi_{y}=L_{X}, y / L_{X}, S \\
& \phi_{f}+\phi_{y}=1
\end{aligned}
$$

where $\boldsymbol{P}_{X, s}$ and $\boldsymbol{P}_{X, f}$ are tensile forces of the sewn specimen and of the fabric element in $\mathrm{X}$ direction, $P_{X, y}(t)$ and $\varepsilon_{X, y}(t)$ being the tensile force and the strain of the seam element in the direction perpendicular to the seam line, respectively. $\phi_{f}$ and $\phi_{y}$ are length fractions of the fabric element and of the seam element in $\mathrm{X}$ direction. In practice, $L_{X, y}$ is made two times as thick as the apparent thickness of the fabric element.

When the model is stretched in $Y$ direction, the tensile force of the sewn specimen $P_{Y, S}$ may be written by the following equations, assuming the simple additive property of the tensile force of the fabric element $P_{Y, f}$ and of the seam element $P_{Y, y}(l)$.

$$
\begin{aligned}
& \varepsilon_{Y}, s=\varepsilon_{Y, f}=\varepsilon_{Y, y}(i) \\
& P_{Y, s}=\phi_{f} \circ P_{Y, f}+\phi_{y} \circ P_{Y, y}(l)
\end{aligned}
$$

where $P_{Y, y}(l)$ and $\varepsilon_{Y, y}(l)$ are the tensile force and the strain of the seam element in the direction paralled to the seam line, respectively.

In the tensile experiment in $\mathrm{Y}$ direction, because the simple attachment assures that $\varepsilon_{X, s}=0, \phi_{f}$ and $\phi_{y}$ can be kept constant. Therefore, this unique experimental method is useful to analyse the tensile behavior of sewn goods when stretched in the direction parallel to the seam line.

Now, consider the case where the cylindrically sewn specimen is stretched perpendicular to the seam while its stitch interval is kept unchanged. For such experiments, the test specimen was prepared by the following way. First, a cylindrically sewn specimen shown in Fig. 3(a) was cut at the middle into two pieces having the same lengths, and then these pieces are sewn together with open-lock stitches along the circumferential line as shown in Fig. 4(a). To eliminate the influence of the seam allowances, these seam allowances are cut at the position shown in Fig. 4(a). 


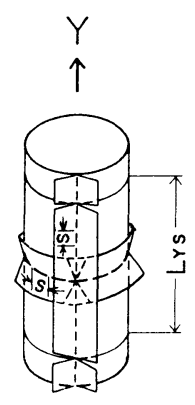

(a)

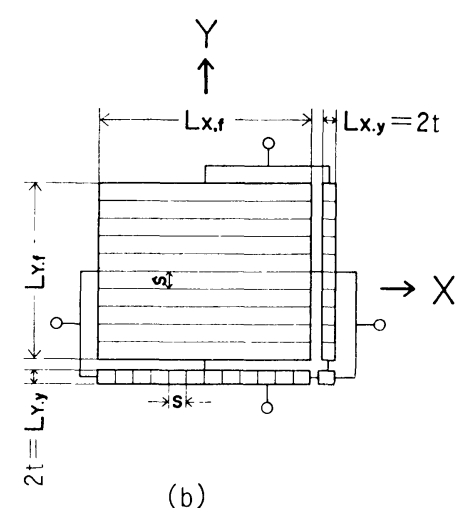

(b)

Fig. 4 (a) Cylindrical by sewn specimen having two seam lines

(b) Equivalent mechanical model

Now consider the bi-axial tensile behavior of the sewn specimen having two seam lines as shown in Fig. 4(a). If n-pieces of mechanically equivalent models are considered as shown in Fig. 4(b), in which the fabric elements and the seam elements are connected partly in parallel and partly in series, we have:

$$
\left.\begin{array}{l}
L_{Y, S}=L_{Y, f}+L_{\bar{Y}}, y \\
\lambda_{f}=L_{Y, f} / L_{Y}, S, \quad \lambda_{y}=L_{Y, y} / L_{Y}, s \\
\lambda_{f}+\lambda_{y}=1
\end{array}\right\}
$$

where $\lambda_{f}$ and $\lambda_{y}$ are length fractions of the fabric and the seam element, and the length of the seam element $L_{Y, y}$ is equal to $2 \mathrm{t}$ in $\mathrm{Y}$ direction. By considering length fractions $\phi_{f}, \phi_{y}$ in eq. (2), the tensile loads and strains in $\mathrm{X}$ and $\mathrm{Y}$ directions are:

In $\mathrm{X}$ direction,

$$
\left.\begin{array}{c}
\varepsilon^{\prime} X, s=\varepsilon_{X}, y \\
P^{\prime} X, s=\lambda_{y} \circ P_{X}, y(l)+\lambda_{f} \circ \varepsilon_{X} \circ P_{X, f}+\phi_{y} \circ \varepsilon_{X}, y(t) \\
=\lambda_{y} \circ P_{X, y}(l)+\lambda_{f} \circ P_{X, y}(t)
\end{array}\right\}
$$

In $\mathrm{Y}$ direction,

$$
\left.\begin{array}{rl}
P_{Y, S}^{\prime} & =P_{Y, y}(t)=\phi_{f} \circ P_{Y, f}+\phi_{y} \circ P_{Y, y}(l) \\
\varepsilon_{Y, S}^{\prime} & =\lambda_{f} \circ \varepsilon_{Y, f}+\lambda_{y} \circ \varepsilon_{Y, y}(t) \\
& =\lambda_{f} \circ \varepsilon_{Y, y}(l)+\lambda_{y} \circ \varepsilon_{Y, y}(t)
\end{array}\right\}
$$

When the attachment shown in Fig. 1 is employed in the experiment, we have $\varepsilon_{X, s}^{\prime}=0$. So, if we have drawn $P_{Y, s}-$ $\varepsilon_{Y, s}$ curve of the sewn specimen shown in Fig. 3(a), the tensile behavior, in the direction perpendicular to the seam line, can be analysed while keeping the original stitch interval.

As is explained in the previous paper ${ }^{[2]}$, the values of $\phi_{f}, \lambda_{f}$ are extremely larger than those of $\phi_{y}, \lambda_{y}$. These facts suggest that the tensile behavior of the sewn specimen solely depends on the behavior of the fabric element until the tensile rupture occurs either within the fabric element or within the seam element. Therefore, if the mechanical

properties of the fabric element are not damaged by the needle action in sewing, the results of the bi-axial tensile experiment and the theories by Kawabata and Niwa et al. ${ }^{[2]}$ can be applied to the tensile results of the fabric element of the sewn specimen.

Now consider the tensile behavior of the seam element when the element is connected with the fabric element. The seam geometry of lock stitches consisting of both a needle-thread and a bobbin-thread varies with the sewing conditions as shown in Fig. 5. In the case of constant fabric feed the relationship between the needle thread tension and the bobbin thread tension exerts much influence on the seam geometry. Fig. 5(a) shows a balanced state of these two tensions, Fig. 5(b) showing the case where the needle thread tension is higher than the bobbin thread tension. As can be seen in Fig. 5(b), the angle at the yarn crossing point is larger with the high tension side than with the low tension side.

From the comparison of Fig. 5(a) with (c), which also shows balanced state of two thread tensions, it is found that the angle at the yarn crossing point decreases as the stitch interval decreases. As the seam geometry varies with the sewing conditions as above mentioned, we shall adopt the geometry shown in Fig. 5(b) as a normal state, and express this seam element with a polygon, shown in Fig. 6(a), which consists of six straight threads, and the lengths and

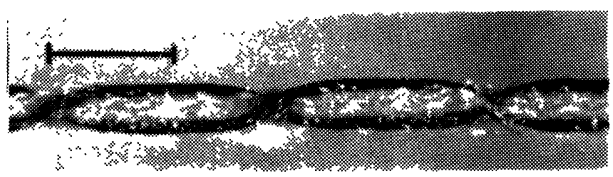

(a)

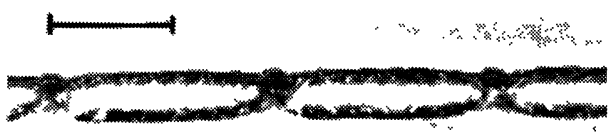

(b)

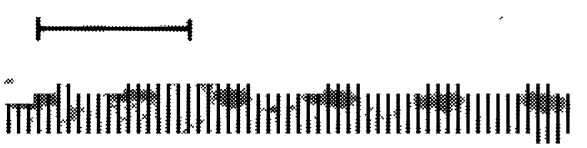

(c)

Fig. 5 Lock stitch loops cut along the seam line. Black bar is $2 \mathrm{~mm}$ long. loop is needle thread.

(a): Balanced seam

(b): Unbalanced seam

(c): Balanced seam with a small stitch interval 


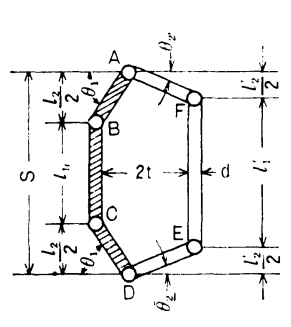

(a)

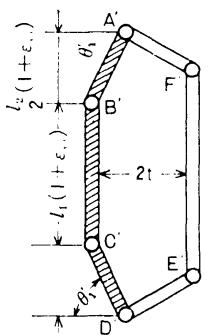

(b)

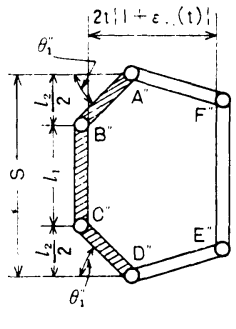

(c)
Fig. 6 (a): Equivalent model of original seam element

(b): Model of seam element stretched paralell to the seam

(c): Model of seam element stretched perpendicular to the seam

the interseting angles of which are illustrated there. When the seam element in Fig. 3(b) is stretched by the same strain as the fabric strain in $\mathrm{Y}$ direction, the lateral length of the seam is kept constant, and is $2 t$. The stretched seam model is shown in Fig. 6(b), in which it is equal to the strain of the fabric element in $\mathrm{Y}$ direction. Thus each strain is given by:

$$
\varepsilon_{Y, f}=\frac{\Delta l_{1}}{l_{1}}=\frac{\Delta l_{2} / 2}{l_{2} / 2}=\frac{\Delta l_{1}{ }^{\prime}}{l_{1}{ }^{\prime}}=\frac{\Delta l_{2} / 2}{l_{2}{ }^{\prime} / 2}
$$

Assuming that eq. (7) is true, each tension differs among six threads. So a tension distribution is introduced in the seam element. Accordingly if each length of six threads and each angle at the yarn crossing point before strain are known, the load-elongation curves of six threads in $\mathrm{Y}$ direction may be obtained by successive calculation, using the load-strain curve of the sewing thread. If tensile rupture occurs within the seam element, the thread having the minimum elongation at break breaks.

In order to find the breaking point in the sewn specimen, it is necessary to compare the elongation at break of the fabric element having the original length of $l_{2}{ }_{2} / 2$ with the minimum elongation at break of six threads recalculated in $\mathrm{Y}$ direction.

Next, consider the deformation of the seam in the direction perpendicular to the seam line as shown in Fig. 6(c). When the seam line on the circumference shown in Fig. 4(a) consists of $\mathrm{m}$ seam elements, and if tensile load $P_{Y, s}^{\prime}=P_{Y, y}^{\prime}(t)$ is applied to the seam element evenly, we have:

$$
P_{Y, y}(t) / m=p_{y}(t)
$$

If $p_{y}(t)$ is applied on $l_{1}$ and $l_{2}$ uniformly, the straight threads of $\overline{\mathrm{AB}}, \overline{\mathrm{CD}} \overline{\mathrm{AF}}$ and $\overline{\mathrm{DE}}$ are stretched in $\mathrm{Y}$ direction, although the yarn tension differs among each thread.

If $\phi_{f} \gg \phi_{y}$ and $P_{Y, y}(t) \doteqdot P_{Y, f}$ in eq. (6), we can predict

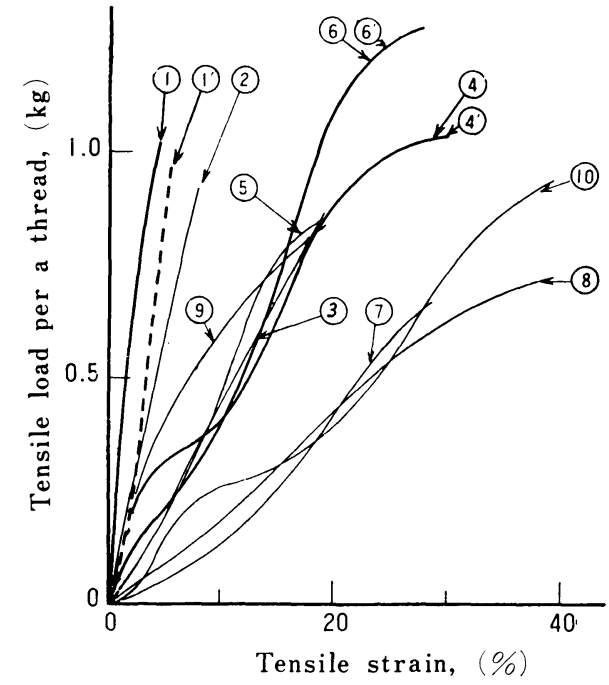

Fig. 7 Tensile load-strain curves of industrial sewing threads at $20^{\circ} \mathrm{C}, 65 \% \mathrm{R} . \mathrm{H}$. S and $\mathrm{F}$ mean spun and filament yarn, respectively.

(1): cotton sewing thread No. 50, waxed, S

(1)': thread (1) after sewn

(2): cotton sewing thread No. $50, \mathrm{~S}$

(3): Polyester-cotton sewing thread No. 50, S

(4): Polyester sewing thread No. 50, F

(4)': Thread (4) after sewn

(5): 6-nylon sewing thread No. $50, \mathrm{~F}$

(6): 66-nylon sewing thread No. $50, \mathrm{~F}$

(6)': Thread (6) after sewn

(7): Crimped nylon sewing thread $110 \mathrm{~d} / 2, \mathrm{~F}$

(8): Monofilament 6-nylon sewing thread No. 50, F

(9): Silk sewing thread No. 50, F

(10): Polyester sewing thread No. 50, S

which element will be ruputured, by comparing the tensile load at break per $\mathrm{S}$ of the fabric element with that of $\overline{\mathrm{AB}}$ or $\overline{\mathrm{AF}}$ thread in $\mathrm{Y}$ direction.

\section{Test Samples}

Because many bi-axial tensile tests have been carried out with various fabrics ${ }^{[2]}$, we put more emphasis, in this experiment, on the seam element than on the fabric element.

\subsection{Sewing threads}

Fig. 7 shows the load-strain curves of commercially available sewing threads pre-conditioned at $20^{\circ} \mathrm{C}, 65 \%$ R.H. for 1 day before tensile experiments. Each curve is selected as a representative having as near value as possible to the arithmetic mean value of ten experiments done at the stretch rate of $200 \% / \mathrm{min}$ in the atmosphere of $20^{\circ} \mathrm{C}, 65 \%$ R.H.

In the sewing process, the needle thread is known to be subjected to repeated tension having nearly the same maximum value before it is formed into a seam ${ }^{[3]}$. On the 
other hand, the tension of a bobbin thread is difficult to measure, and the precise knowledge of it is still left to be explored.

However, the tension of the bobbin thread is governed by the friction between the thread and the metal surface of the regulator as in the case of the needle thread. For that reason, it is supposed that the bobbin thread, until it is formed into a seam, is subjected to a repeated load having different frequency from that of the needle thread.

In order to know the tensile behavior of threads after repeated loading, load-strain curves were measured with yarns (1), (4), and (6) after they were removed from the sewn specimens, and shown in Fig. 7 with '. Although the curve of spun yarn (1') is slightly different from that of (1), curves of filament yarns (4') and (6') are much similar to those of (4) and (6), respectively.

With threads (4) and (6), the maximum tension in sewing is assumed to be smaller than the yield stress of the thread ${ }^{[3]}$.

With thread (1), the strength decrease may be because of the disturbance of fiber orientation by both the repeated stretch before sewn-in and friction when passing through the fabric.

\subsection{Fabric}

In this experiment, a plain woven fabric, of low tensile strain at break was used. Its structural characteristics are listed in Table 1.

\section{Tensile Experiment of Each Element and of Cylindrical Specimens}

5.1 Experiment of the seam element

Tensile behavior of a seam approximated as shown in Fig. 6(a) was observed by using an attachment described before. For stretching in the direction parallel or perpendicular to the seam line, the sewing thread was mounted on the attachment as shown by either the two-dotted line or the solid line, in Fig. 8, which corresponds to BAF of $\theta_{1}=\theta_{2}$ or DCBA in Fig. 6(a). In the latter case, points $\mathrm{B}$ and $\mathrm{C}$ in Fig. 6 correspond to fixed rods (5) in Fig. 8. So we changed the rod material as follows to realize the different connecting conditions: i) Teflon, ii) metal covered with No. 100 emery paper, iii) clamps instead of rods.

Fig. 9 shows the experimental results thus obtained. In case of i), since the frictional coefficient between Teflon rods and the thread is low, the thread slips on the rod to shift the load-strain curve to a high strain side. Curves for case ii) fall roughly in the middle between case i) and case iii). Threads made of synthetic fibers are broken at the point contacting with the rod, to give low strength at break.

Curves for case iii) are identical to those of the tensile experiment of BAF done in the direction parallel to the seam line.

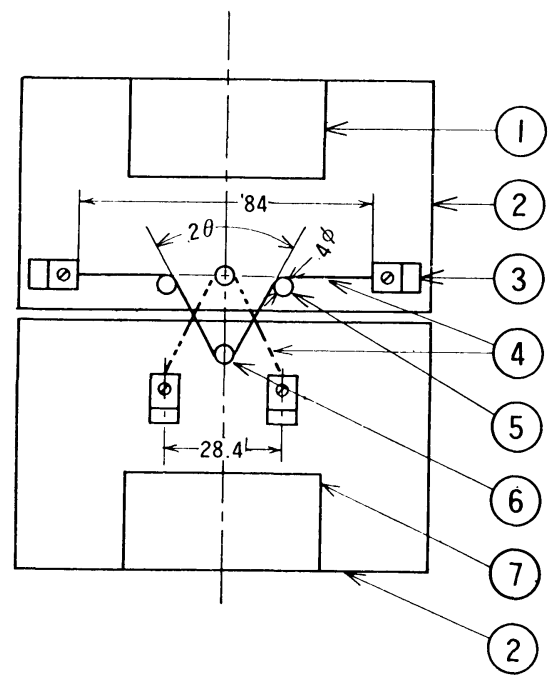

Fig. 8 Attachment employed in tensile experiment of the model seam element.

(1), (7): upper and lower clamps.

(2): base plate, (3): clamps for sewing thread.

(4): sewing thread, (5): fixed rods, (6): rod for applying load

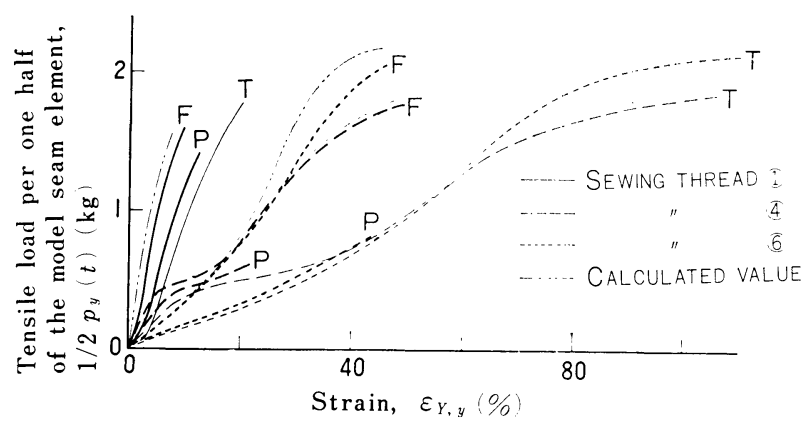

Fig. 9 Tensile load-strain curves for seam element ABCD shown in Fig. 6. Load is applied in the direction perpendicular to the stitch length. $2 \theta$ is $80^{\circ}$.

F: When fixed at B, C. shown in Fig. 6

P: With emery paper on the fixed rod

$\mathrm{T}$ : With a fixed rods made of Teflon

If eq. (7) is held, a small decrement of the angle at the yarn crossing point can be written as $\tan \left(\theta_{2}+\mathrm{d} \theta_{2}\right)=$ $\left(l^{\prime}{ }_{2} / 2+\Delta l^{\prime}{ }_{2}\right) / t$. So, the force component in $\mathrm{Y}$ direction of B'A' $F$ ' in case of $\theta=40^{\circ}$ can be calculated by using tensile load-strain curves of sewn yarns in Fig. 7, and the results are shown in two dotted lines in Fig. 9. These curves are in close agreement with the curves obtained in case of iii).

\subsection{Experiment of a fabric element}

To obtain $P_{Y, f}-\varepsilon_{Y, f}$ curves of the fabric element alone, a bi-axial tensile experiment was carried out by using the fabric formed into a circular cylinder having many knotts 


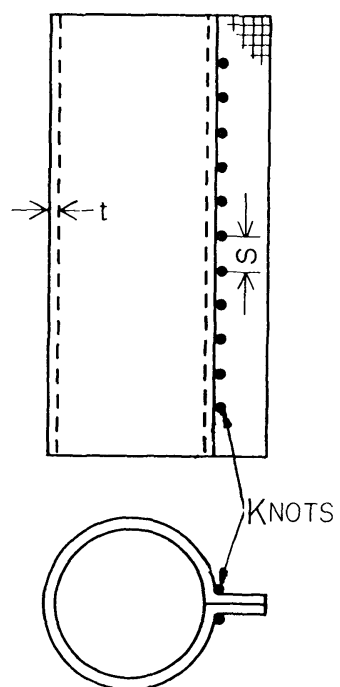

Fig. 10 Schematic diagram of a fabric element for bi-axial testing

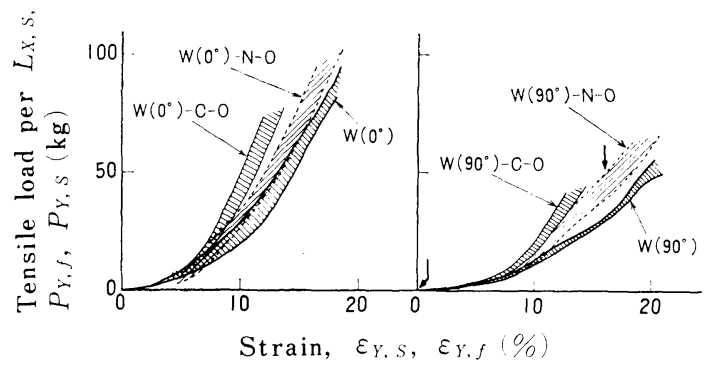

Fig. 11 Load-strain curves of fabric elements and sewn specimens shown in Fig. 10 and Fig. 3 (a)

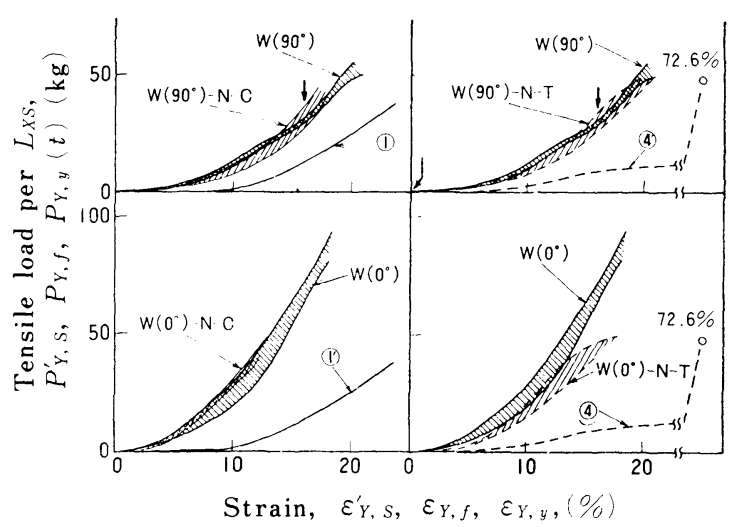

Fig. 12 Load-strain curves of fabric elements, seam elements and the sewn specimens shown in Fig. 4 (a)

at the points corresponding with stitch holes on the seam line as shown in Fig. 10. $P_{Y, f}-\varepsilon_{Y, f}$ curves are shown in Figs. 11 and 12.
5.3 Experimental results of lock stitched fabrics

Sewn specimens made in the following way and listed in Table 2 were used.

The picce, $18 \times 18 \mathrm{~cm}$ wide, was sewn to fit to the Teflon rod of $35.6 \mathrm{~mm}$ in diameter, with seam allowance of more than $2 \mathrm{~cm}$, under the sewing condition that the seam geometry shown in Fig. 5(a) is obtained. Stitch intervals are $1.2,2.7$ and $3.6 \mathrm{~mm}$. To prevent the tensile rupture in the seam line parallel to the generating line, the specimen shown in Fig. 4(a) was sewn of thread (6).

After sewing, specimens were kept at $20^{\circ} \mathrm{C}, 65 \%$ R.H. for about one day, and cut in $120 \mathrm{~mm}$, in length to be stretched at a tensile rate of $10 \mathrm{~mm} / \mathrm{min}$ under the atmospheric condition above mentioned.

Experimental results of specimens having the stitch interval of $3.6 \mathrm{~mm}$ are shown in Figs. 11 and 12. In these figures, letters $\mathrm{W}\left(0^{\circ}\right)$ and $\mathrm{W}\left(90^{\circ}\right)$ mean the results of the fabric element alone stretched in the warp and in the weft directions, respectively. Variation of measured results is due to the change of the number of yarns per $L_{X, s}$, and is expressed by the hatched region in Figs. 11 and 12. Fig. 11 shows that all the curves of sewn specimens are nearer to the load axis than curves of fabric elements alone owing to the influence of sewing threads. Namely, in case of $\mathrm{W}\left(0^{\circ}\right)$ $\mathrm{C}-\mathrm{O}$ and $\mathrm{W}\left(90^{\circ}\right)-\mathrm{C}-\mathrm{O}$, the rupture takes place at the seam element having low elongation at break. Consequently, the strength at break of the sewn specimen is lower than that of the fabric element alone. When nylon 66 sewing thread having large elongation at break is used, sewn specimens are rather stronger than the fabric element alone, and the rupture occurs frequently at fabric elements.

Fig. 12 shows load-strain curves of the sewn specimen calculated by both the load-strain curves of sewing threads and the seam geometry shown in Fig. 5(a). Circumferencial rupture occurs with $\mathrm{W}\left(90^{\circ}\right)-\mathrm{N}-\mathrm{C}, \mathrm{W}\left(0^{\circ}\right)-\mathrm{N}-\mathrm{C}$ and $\mathrm{W}\left(0^{\circ}\right)$ $\mathrm{N}-\mathrm{T}$, and the strength at break of sewn specimens are nearly equal to that of the seam element alone. As seen in the upper right side in Fig. 12, the load at break of W $\left(90^{\circ}\right)$ is roughly equal to that of the seam element sewn of thread (4). However, compared with the strength of $\mathrm{W}\left(90^{\circ}\right)-\mathrm{N}-\mathrm{O}$ in Fig. 11 with that of the seam element, the latter is lower than the former.

Fig. 13 shows the cloth appearance of some seam elements with no strain and with strain at the level pointed with arrows in Figs. 11 and 12. Even when W(90)-N-O specimen was stretched to $16 \%$ elongation, which is nearly equal to the strain at break, the deformed fabric element lying a little bit apart from the seam line looks like the deformed fabric near the seam line. Therefore, we assumed that, as shown in Fig. 6(b), the strain at each portion of the seam element is given by eq. (7).

Comparing two figures of $\mathrm{W}\left(90^{\circ}\right)-\mathrm{N}-\mathrm{T}$, the stitch interval 

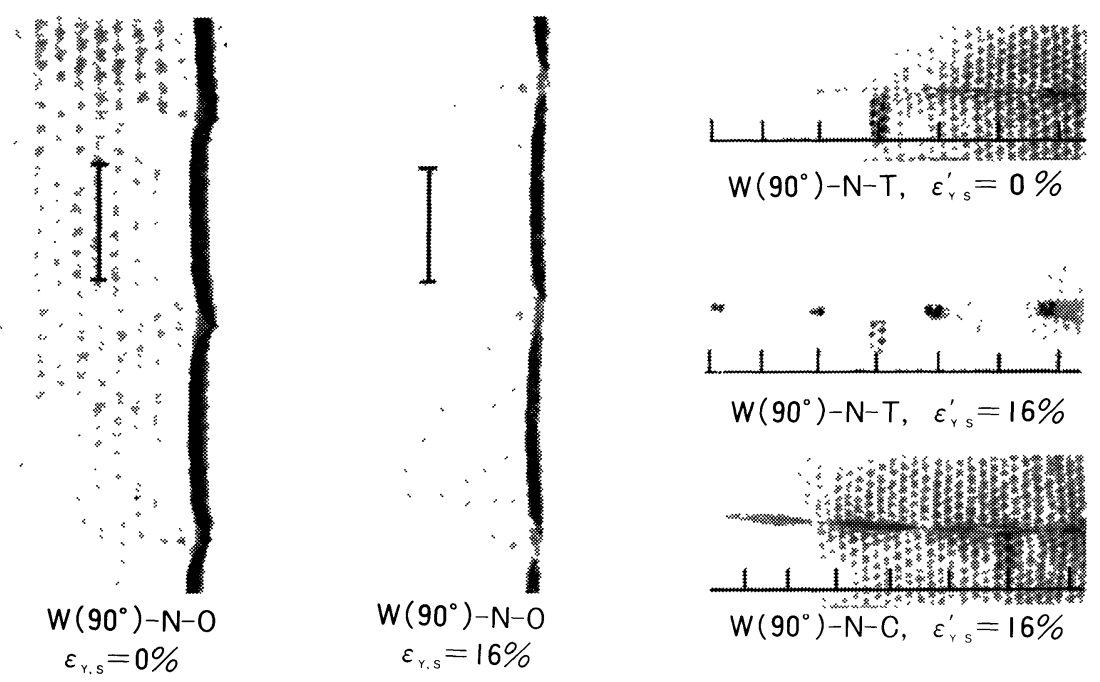

Fig. 13 Cloth appearance before and after stretched. One unit of the scale corresponds to $2 \mathrm{~mm}$ in length.

at $\varepsilon_{Y . s}^{\prime}=0 \%$ is equal to that at $\varepsilon_{Y . s}^{\prime}=16 \%$. The result supports that our experiment was done strictly in the biaxial extensions of sewn specimens.

On the circumferencial seam element sewn of different threads, even when the same strain as $\varepsilon_{Y, s}^{\prime}=16 \%$ is applied to $\mathrm{W}\left(90^{\circ}\right)-\mathrm{N}-\mathrm{T}$ or $\mathrm{W}\left(90^{\circ}\right)-\mathrm{N}-\mathrm{C}$, the former shows seam grins as shown in Fig. 13, resulting in seam damage. Judging from these deformed states of $\mathrm{W}\left(90^{\circ}\right)-\mathrm{N}-\mathrm{T}$ and $\mathrm{W}\left(90^{\circ}\right)$ $\mathrm{N}-\mathrm{C}$ the mechanical model shown in Fig. 4(b) may be right and sound.

\section{Discussion}

When the stitch interval $\mathrm{S}$ and the angle at the yarn crossing point $\theta\left(\theta=\theta_{1}=\theta_{2}\right)$ in Fig. 6(a) are changed, the loadstrain curves in $\mathrm{Y}$ direction are calculated by using loadstrain curves of (1'), (4') and (6') in Fig. 7 and load-strain curves of fabric element alone in Fig. 11 are shown in Fig.



Fig. 14 Load-elorgation Corves of the seam slement and the falric element.



Fig. 15 Tensile load-elongation curves of the fabric and the seam elements when stitch interval $\mathrm{S}$ is varied

14. The tensile load per $2 \mathrm{t}$ and the elongation per $l_{1}$ or $l_{2} / 2$ are also shown in Fig. 14. Comparison of these curves shows that the tensile rupture of sewn specimens occurs at the part having the lowest elongation at break. For example, specimens, sewn of thread (1) and of a stitch 
interval of $3.6 \mathrm{~mm}$, break at $\mathrm{AB}$ of the seam element. But specimens, sewn of thread (4) or of (6), break at the fabric element.

Relationship betwen the tensile load per s and the elongation of the seam element is shown in Fig. 15 with three kinds of sewing threads. It shows that the tensile rupture of these sewn specimens occurs at the part having the lowest strength at break. When the stitch interval is high and $3.6 \mathrm{~mm}$, specimens sewn of threads (1), (4) or (6) and with fabric $\mathrm{W}\left(0^{\circ}\right)$, and specimens sewn of threads (1) and with fabric $\mathrm{W}\left(90^{\circ}\right)$, break at the seam element. But specimens sewn of thread (4) or (6) and with fabric $\mathrm{W}\left(90^{\circ}\right)$ show first the seam grin, and then often break at the seam element.

When the stitch interval is low and $1.2 \mathrm{~mm}$, the load at break of the fabric elements decreases per $\mathrm{S}$, but that of the seam element increases due to the decrease of the angle at the yarn crossing point. So, in this case, the fabric element breaks at first. It is generally known ${ }^{[4]}$ that the tensile strength in the direction perpendicular to the seam line of a sewn specimen increases first with increasing the stitch density, and then stays constant in the high stitch density range. Compared this fact with our experimental results, it follows that: the increase of the tensile strength in the above mentioned range is due to the increase of seam strength in $\mathrm{Y}$ direction owing to the decrease of the stitch interval; in the high stitch density range the tensile rupture of sewn specimens is observed in the fabric element.

\section{Conclusion}

Woven fabrics were sewn to form cylinders into which a Teflon rod was inserted to prevent the cylinder diameter from being diminished. The fabrics were then stretched along the axis of the Teflon rod, and the resuits were analysed by using mechanically equivalent models. It was obtained that, when fabrics are sewn together, it is necessary to increase the seam strength by balancing both tensions of the needle thread and the bobbin thread.

\section{Literature cited}

[1] S. Kawanishi and T. Horino, J. Text. Mach. Soc. Japan, 24, T85 (1971)

[2] M. Niwa and S. Kawabata, ibid, 23, T85, T133, T257 (1969)

[3] T. Horino and S. Kawanishi, J. Japan Res. Ass. Text. End-Uses, 10, 2 (1969)

[4] For example, "Saishin Hifukugaku", Chapt. 3, published by The Textile Machinery Sosiety of Japan, 1968 or "Saishin Hosei Kagaku", Chapt. 3, published by The Japan Research Association For Textil Eend-Uses (1969) 